\title{
Enhanced Confocal Microscopy Imaging of the In-Plane Switching of Cholesteric Liquid Crystal Cells
}

\author{
S. A. Jewell*a J. R. Sambles ${ }^{\mathrm{a}}$ \\ ${ }^{a}$ Electromagnetic Materials Group, School of Physics, University of Exeter, \\ Stocker Road, Exeter, EX4 4QL, United Kingdom
}

\begin{abstract}
The recently developed fluorescence confocal polarizing microscopy (FCPM) imaging technique allows 3D images of the director structure in a liquid crystal cell to be resolved with sub-micron resolution. Results are presented on imaging the response of 5-micron pitch cholesteric liquid crystals to an in-plane electric field applied between two silver electrodes. The results show, in exquisite detail, how the application of an in-plane field causes the cholesteric helix to tilt through $90^{\circ}$ either within, or immediately adjacent to, the electrode gap depending on the sign of the dielectric anisotropy of the liquid crystal. Furthermore, imaging the cholesteric material above the silver electrodes reveals a previously unreported optical intensity enhancement. This phenomenon is discussed along with its possible benefits to the existing imaging technique. The effects of the point spread function of the system are discussed and a ray optics model is used to produce model data highlighting the influence of this phenomenon on the recorded results.
\end{abstract}

Keywords: Fluorescence confocal microscopy, liquid crystals, cholesteric, in-plane switching.

\section{INTRODUCTION}

Liquid crystal displays controlled by in-plane electric fields are becoming increasingly common and offer advantages such as improved viewing angle and optical contrast when compared to conventional twisted nematic liquid crystal devices. In such devices transparent electrodes separated by a few microns are deposited on the back-plate of the display and the liquid crystal alignment is controlled by applying a potential difference between them. Studies of commercially significant displays including those using in-plane switching (IPS) [1] and fringe field switching (FFS) [2] show that the successful devices depend upon the optimization of many cell parameters. These include the initial alignment direction, electrode width, and electrode gap [3] along with liquid crystal parameters such as the dielectric anisotropy, elastic constants and the rotational viscosity coefficient [4]. As the demand for improved resolution and miniaturized pixels in displays grows the influence of stray switching fields on adjacent pixels becomes increasingly problematic and is an area that needs careful consideration. This phenomenon is of particular interest as analysis of the viewing angle characteristics of FFS and IPS devices have shown that these fields actually contribute to their switching characteristics [2].

The ability to resolve in detail the complex effect of these fields on the director profile has, until recently, been extremely limited. Standard polarizing microscopy techniques allow distortions within a liquid crystal sample to be observed through the inherent birefringence of the material [5] but the resulting image is an integration of the light transmitted through the cell and so any depth information is lost in the 2D image produced. Powerful optical waveguide characterisation techniques have previously been used to determine the director profile in both static $[6,7]$ and dynamic $[8,9]$ liquid crystal alignments. However, the relatively large beam-spot used $(200 \mu \mathrm{m})$ and the transit of the incident beam through the entire thickness of the sample restricts such techniques to uniform samples which vary in only one dimension. However, with the advent of micron-scale surface relief structures for use in low power bistable display applications such as zenithal bistable devices [10] and post-aligned bistable nematic displays [11] the director distortion around these features can occur in two or even three dimensions. As a result, to allow a detailed understanding of the behavior of the liquid crystal in such devices it is important to develop measurement techniques which can allow the direct characterisation of the liquid crystal director and accommodate these added spatial dimensions.

\subsection{Fluorescence Confocal Polarizing Microscopy (FCPM)}

Fluorescence Confocal Polarizing Microscopy (FCPM) is a 3D optical imaging technique that has been successfully used in many biological studies over the past two decades [12]. The process involves doping the sample of interest with a low 
concentration of a fluorescent dye which is preferentially absorbed by the structure of interest within the specimen. The dye has a high quantum yield, absorbing strongly at one wavelength and emitting at one of a higher value. By recording the fluorescence produced when the sample is probed with light at the excitation wavelength at various points through the sample a 3D image of the fluorescence and hence the distribution of the structure of interest is produced. It has been shown that this method can be adapted to probing the alignment of low-birefringence liquid crystals by using an anisomeric dye molecule $[13,14]$. For a suitably low concentration $(\sim 0.01 \%$ by weight $)$ the dye orients with the liquid crystal director (average local molecular orientation) without significantly perturbing the alignment or liquid crystal properties, and the dye molecular alignment follows the director through the cell. For a linearly polarized excitation beam the efficiency of the absorption of the dye and the fluorescence intensity of the emitted light is controlled by the angle $\theta$ between the polarization axis of the incident light and the absorption axis of the dye molecule, with the fluorescence intensity being proportional to $\cos ^{2} \theta$ [15]. The average orientation of the dye molecules, and hence the director in the surrounding region, is imaged by mounting the sample in a confocal fluorescence microscopy set-up (figure 1) and scanning over $\mathrm{a} \sim 100 \mu \mathrm{m} \times 100 \mu \mathrm{m}$ area in the $x-y$ plane at a fixed depth $z$. This produces an optical "slice" of intensity versus position in this plane and by systematically repeating this procedure at a series of fixed depths through the cell, a 3D model of the director orientation is produced. To ensure that only light emitted from the focal region of the confocal beam is collected, a pinhole is used at the focal point of the light emitted from the voxel of interest to allow a spatial resolution of better then $1 \mu \mathrm{m}$ in all three dimensions.

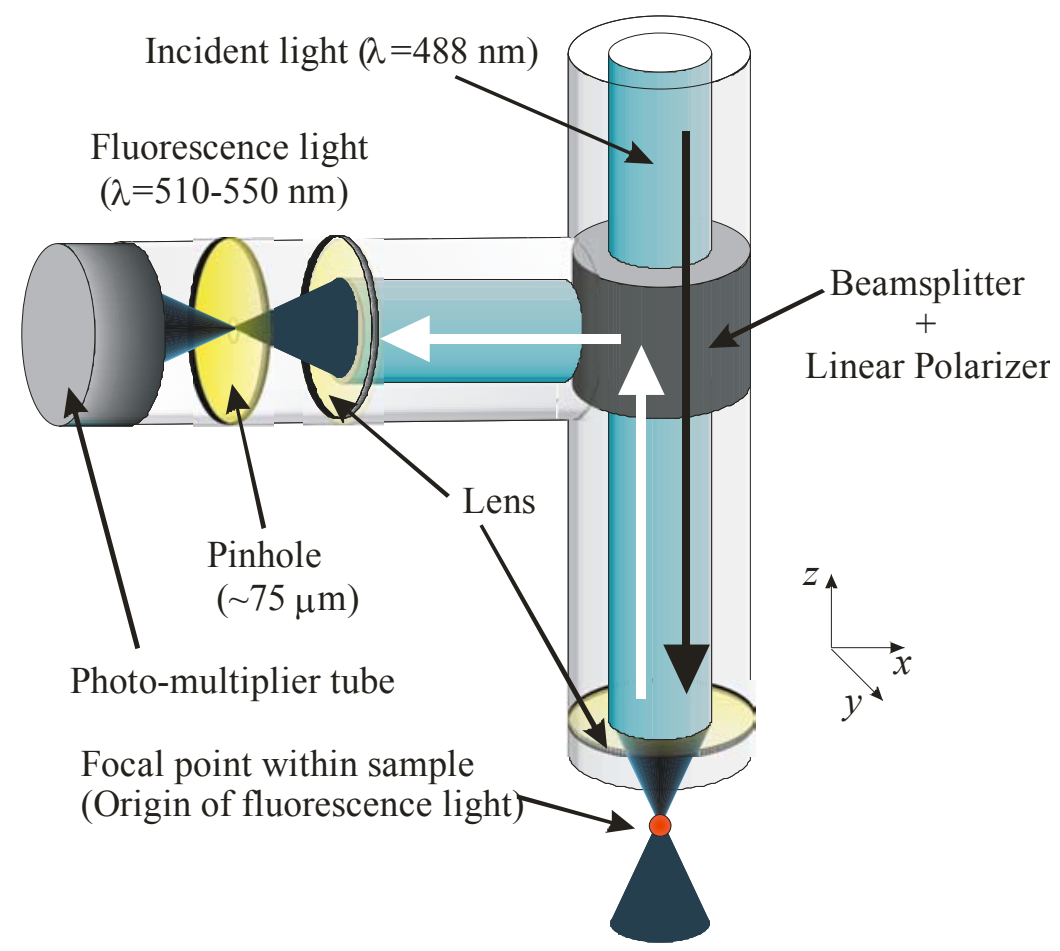

Fig. 1 Schematic diagram of the lens and pinhole arrangement used in the optical set-up of a Fluorescence Confocal Polarizing Microscope. The strong black and white arrows denote the direction of the excitation and emitted light respectively.

This technique has previously been used to examine such structures as twist-grain boundaries in free-standing liquid crystal films [16], defects in biaxial smectic-A materials [17] and focal-conic domains in self-assembled structures [18]. It has also been shown that FCPM can be used to probe the cholesteric structure (of pitch $p$ ) in a Grandjean-Cano wedge cell [19] provided that the parameter $p \Delta n / 2 \lambda$ is small enough to limit the influence of the Maugin effect on the resulting images. 


\section{METHODOLOGY}

\subsection{Cell fabrication}

A number of liquid crystal cells containing in-plane silver electrodes were constructed for use in this study. A $160 \mu \mathrm{m}$ thick plain glass cover- slip $(n=1.52)$ was used as the substrate for the incident face of the cell to aid the focusing of the beam within the sample and reduce losses. The superstrate was formed from a $1.5 \mathrm{~mm}$ thick glass slide $(n=1.52)$. A glass fibre of a thickness equal to the required electrode gap was positioned on the superstrate to act as a mask and an optically thick $(100 \mathrm{~nm})$ layer of silver was thermally evaporated onto the slide. The fibre was then removed and both plates were spin coated with a commercial polyimide (JSR AL1254), baked and rubbed perpendicular to the electrode gap to produce homogeneous alignment. The cell was then assembled using strips of $15 \mu \mathrm{m}$ thick mylar as spacers along two edges and sealed using UV curing glue (figure 2). Two liquid crystals, ZLI-2293 (Merck KGaA, $\Delta n=0.133, \Delta \varepsilon=$ +10 ) and MLC-6610 (Merck KGaA, $\Delta n=0.098, \Delta \varepsilon=-3.1$ ) were doped to a concentration of $\sim 2.7 \%$ by weight with the chiral dopant $\mathrm{CB} 15$ (Merck KGaA) to create a cholesteric pitch of $\sim 5 \mu \mathrm{m}$ in each material and allowed to mix in the isotropic phase overnight. The individual cholesteric mixtures were then doped with a low concentration $(0.01 \%$ by weight) of the fluorescent dye N,N-bis(2,5-di-tert-butylphenyl)-3,4,9,10-perylenedicarboximide (BTBP) and mixed thoroughly. The mixtures were introduced into separate cells and in all cases, examination under crossed polarizers showed that a good cholesteric monodomain was formed. Finally, wires were soldered onto the exposed areas of the silver electrodes to allow the application of in-plane voltages.

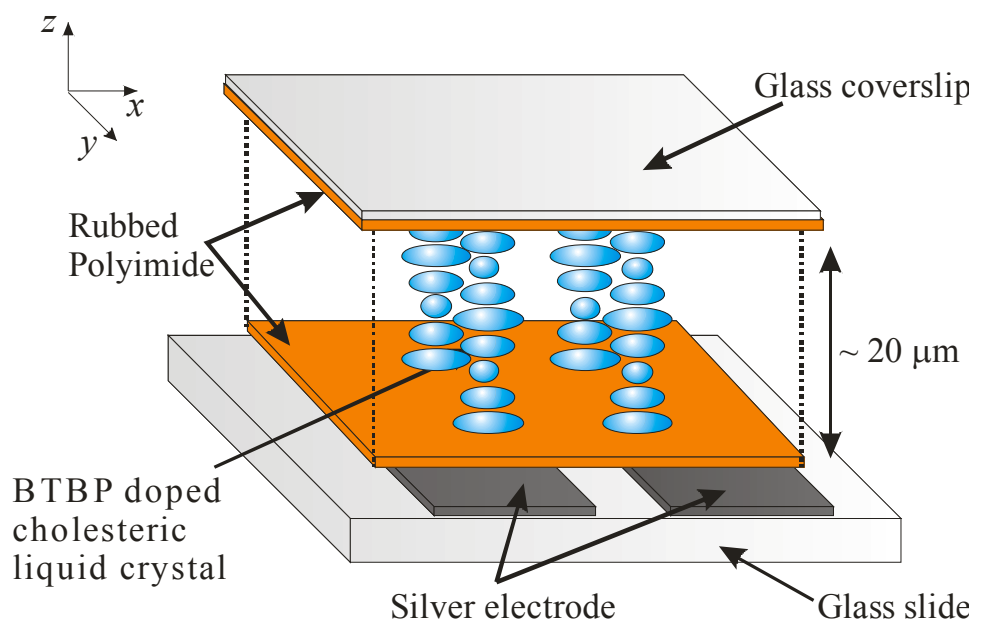

Fig. 2 Schematic diagram of a typical cholesteric liquid crystal cell used in an FCPM study (not to scale).

\subsection{Data collection}

The liquid crystal cells under test were mounted in an inverted fluorescence confocal polarizing microscope system (Leica TCS SP5) operating in reflection mode, with the incident face (cover-slip) index-matched to a $63 \times$ oil immersion lens $(\mathrm{NA}=1.4)$ and the rubbing direction of the cell parallel to the polarization of the incident light. For the fluorescence dye selected (BTBP) an excitation wavelength of $488 \mathrm{~nm}$ was required and the fluorescence light emitted was collected over the range $510-550 \mathrm{~nm}$. FCPM images were obtained over areas in the $x-y$ plane covering a few hundred $\mu \mathrm{m}^{2}$ (depending on the electrode gap used). These scans were repeated in $0.2 \mu \mathrm{m}$ steps through the cell over a total depth of $\sim 40 \mu \mathrm{m}$ in the $z$-direction. The entire scan was then repeated with various $10 \mathrm{kHz}$ a. c. potential differences applied across the electrode gap. To allow the sample to reach equilibrium, it was necessary to leave the sample for at least 10 minutes after the application of each voltage before commencing the scan to allow any defects etc. to dissipate. For each set of data, image reconstruction software (Image $\mathrm{J}$ [20]) was used to compile the in-plane images and construct a crosssection through the cell showing how the fluorescence intensity varied with depth. 


\section{RESULTS AND ANALYSIS}

\subsection{Positive dielectric anisotropy (ZLI-2293*)}

The reconstructed cross-sections in the $x-z$ plane of the ZLI-2293* cell with $0,15,25$ and $50 \mathrm{~V}_{\mathrm{pp}}$ applied across the $25 \mu \mathrm{m}$ electrode gap are shown as grey-scale images in figure 3. The upper $6 \mu \mathrm{m}$ thick black region of each image corresponds to the cover-slip substrate of the cell, which does not fluoresce at the chosen wavelengths. The lower $6 \mu \mathrm{m}$ section corresponds to the glass superstrate of the cell, with the black region corresponding to the area directly below the electrode gap. As discussed later, the remainder of this lower region shows the reflection of incident light from areas above the silver electrode and so gives a false impression of fluorescence light originating from this area. The central $25 \mu \mathrm{m}$ deep region of the image collected with the cell shorted (figure 3(a)) clearly indicates a cholesteric structure of pitch $\sim 5 \mu \mathrm{m}$. This is evident from the dark and light bands, indicating that the fluorescent dye (and hence the director) is periodically either parallel or perpendicular to the polarization of the excitation beam resulting in maximum or minimum fluorescence respectively.

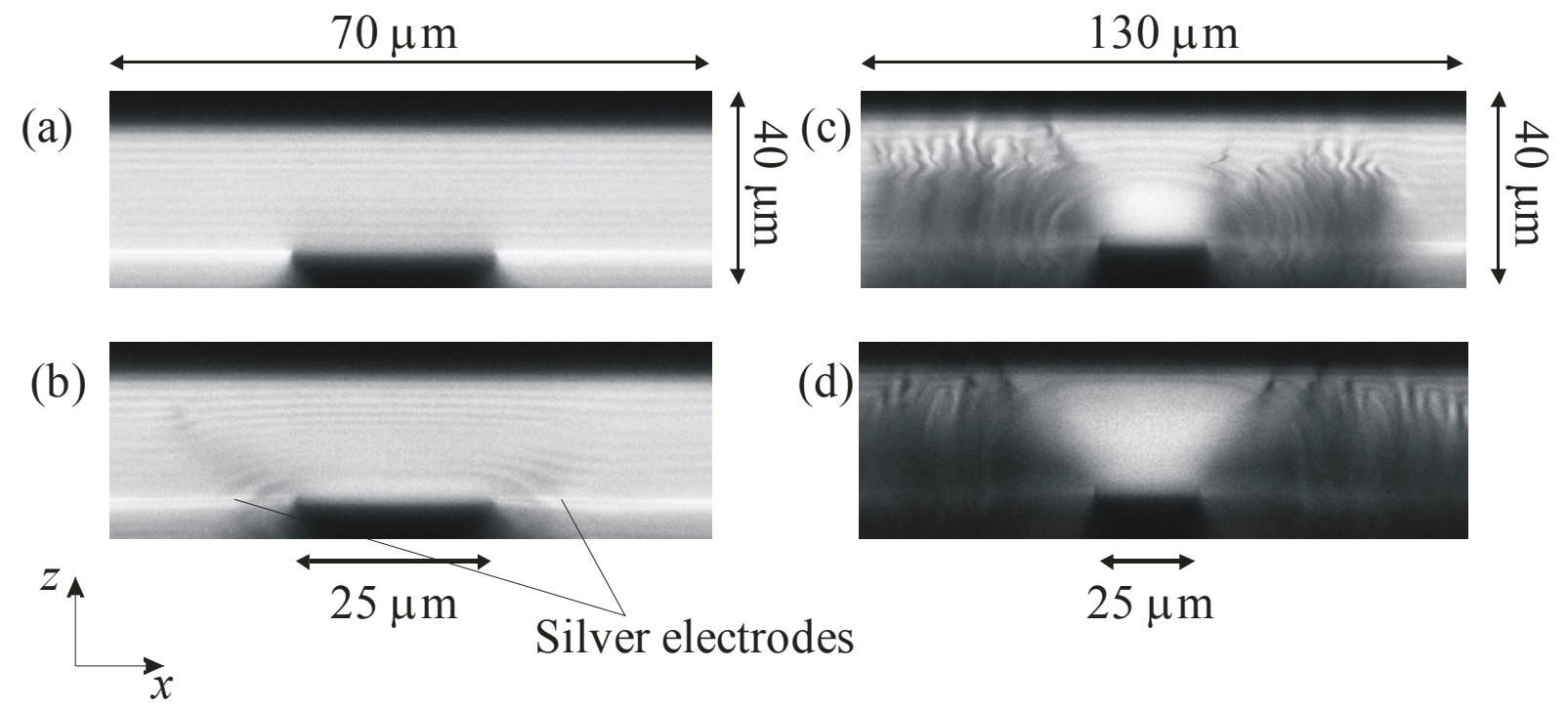

Fig. 3 Cross-section grey-scale images from an x-z plane taken from a ZLI-2293* liquid crystal cell at applied peak-to-peak voltages of (a) $0 \mathrm{~V}$, (b) $15 \mathrm{~V}$, (c) $25 \mathrm{~V}$ and (d) $50 \mathrm{~V}$. The fluorescence intensity is shown as a function of position using a scale varying linearly from black (no fluorescence) to white (maximum recorded fluorescence).

The application of different potential differences across the $25 \mu \mathrm{m}$ electrode gap indicates that two different types of deformation occur. At $15 \mathrm{~V}_{\mathrm{pp}}$ (figure 3(b)) a buckling of the cholesteric layers is first observed in a region which extends outside the electrode gap. In addition, the liquid crystal in the region of the strongest field (i.e. directly above the glass between the electrodes) begins to untwist, aligning with the field (and direction of the polarization of the excitation laser) resulting in an increase in fluorescence intensity, in accordance with predictions from considering weak azimuthal anchoring [21]. As the in-plane voltage is increased the lateral distance over which the buckling occurs increases, and by $50 \mathrm{~V}_{\mathrm{pp}}$ the liquid crystal appears to form a cholesteric structure with the helix lying along the $x$-axis of the system with a $5 \mu \mathrm{m}$ pitch. The clarity of the pitch structure at the higher voltages is blurred due to the continual movements of defects through the region which mediate the helical reorientation over the several minutes duration of the scanning process.

Images collected in the $x-y$ plane, close to the top of the cell during the application of a $50 \mathrm{~V}_{\mathrm{pp}}$ voltage are shown in figure 4. The first was collected with the polarization of the incident beam parallel to the field (and hence director alignment) and the second was collected with the polarization rotated through $90^{\circ}$. The width of the perceived electrode 
gap in these images appears greater than the physical dimensions of the electrode gap $(25 \mu \mathrm{m})$ due to the extended "V" shaped nature of the region within the cell where the helix has been unwound by the applied field (Fig. 3(d)). These images highlight the contrast achievable using the FCPM (measured as a maximum to minimum ratio of 7:1) and also allow some additional information on the alignment of the director in the dark regions of the images. For example, in figure 4(a) the black regions indicate that the director could be either lying homogeneously along the $y$-axis or aligned homeotropically along the $z$-axis. The generally low intensity of the corresponding regions in figure 4(b) discounts the notion of the director lying along the $y$-axis and so confirms that the director is homeotropic. The over-all impression from the low fluorescence intensity in figure 4(b) is that over this region the two main director alignments are along the $x$ - and $z$-axes. The subtle variations in intensity that can be seen are likely to be due to a small angular off-sets between the helix and the polarization direction. In addition, the uniformity of the fluorescence in the regions above the electrode gap in figure 3(d) and the central band in figure 4(a) suggests that the dye is well aligned by the liquid crystal. Due to the negligible dielectric anisotropy of the dye it can be assumed that this alignment is purely due to the influence of the host liquid crystal rather than the field itself.

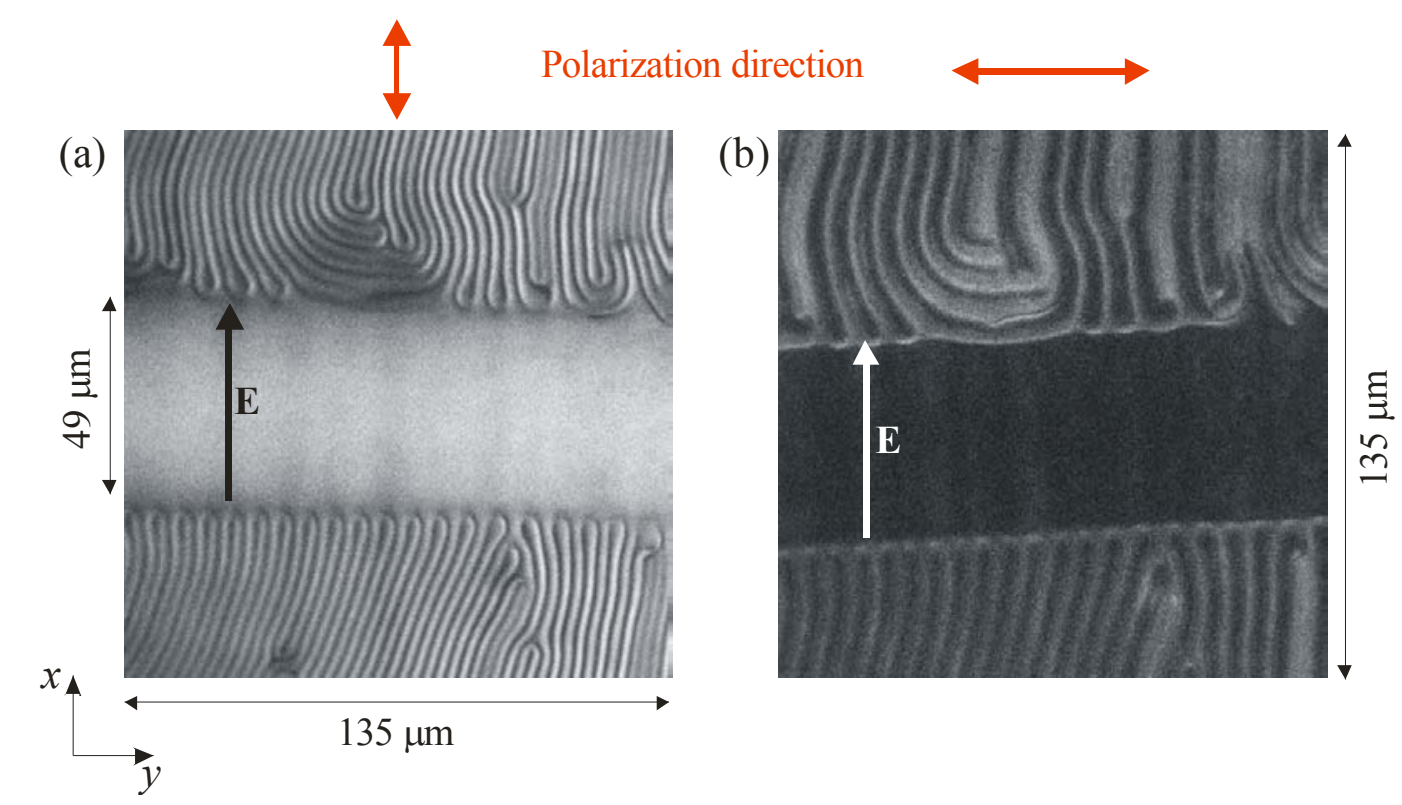

Fig. 4 Grey-scale images from an $x-y$ plane close to the incident surface of a ZLI-2293* liquid crystal cell at an applied in-plane voltage of $50 \mathrm{~V}_{\mathrm{pp}}$. with the incident excitation beam polarization oriented (a) perpendicular and (b) parallel to the electrode gap.

\subsection{Negative dielectric anisotropy (MLC-6610*)}

The result of applying an in-plane field to a cell containing a negative dielectrically anisotropic material (MLC-6610*) is in stark contrast to the effects observed with a positive material. Figure 5 shows that the application of a voltage above a threshold potential of $\sim 30 \mathrm{~V}_{\mathrm{pp}}$ across a $125 \mu \mathrm{m}$ electrode gap initially causes a buckling of the cholesteric layers in the regions closest to the electrode edges, with the $5 \mu \mathrm{m}$ pitch cholesteric structure maintained in the central region of the electrode gap. It was observed that the buckling was mediated by defects seen to move along the $y$-axis, parallel to the electrode edges, over a period of 10 minutes or more. As the voltage was increased, the extent to which the buckling penetrated into the electrode gap increased but, as can be seen from Figure 5(c), the buckling was not necessarily symmetrical across the gap. It is presumed that this was due to the transition being driven by the motion of defects and that the path of these may have been influenced by perturbations along the electrode edges in areas outside the region being scanned. Figure 5(d) shows the result of the sample being allowed to reach equilibrium with $100 \mathrm{~V}_{\mathrm{pp}}$ applied across the electrode gap. It can be clearly seen that the liquid crystal at the centre has formed a $\sim \mu$ m pitch cholesteric structure with the helical axis rotated through $90^{\circ}$ and lying across the electrode gap (i.e. along the $x$-axis) with the extraordinary axis of the liquid crystal lying in the $y-z$ plane, perpendicular to the field. Through adopting this conformation 
both the dielectric and elastic (twist) energies of the system are minimized, along with the surface anchoring constraints through the periodic variation in director alignment at the surfaces.

$185 \mu \mathrm{m}$

(a)

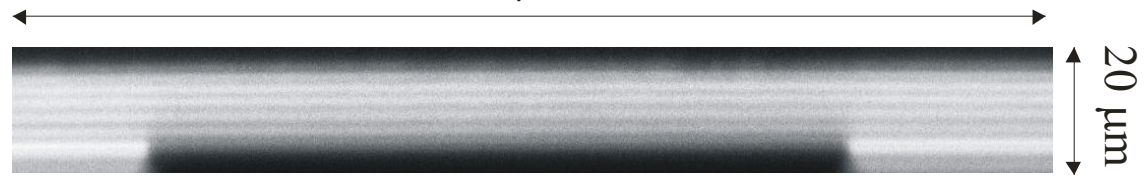

(b)

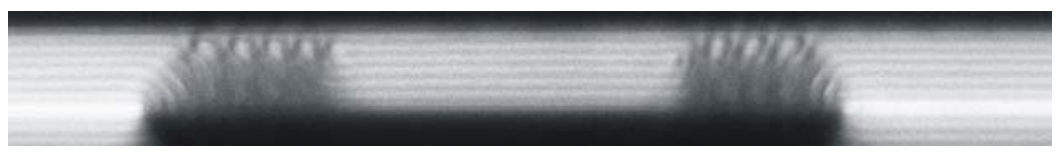

(c)
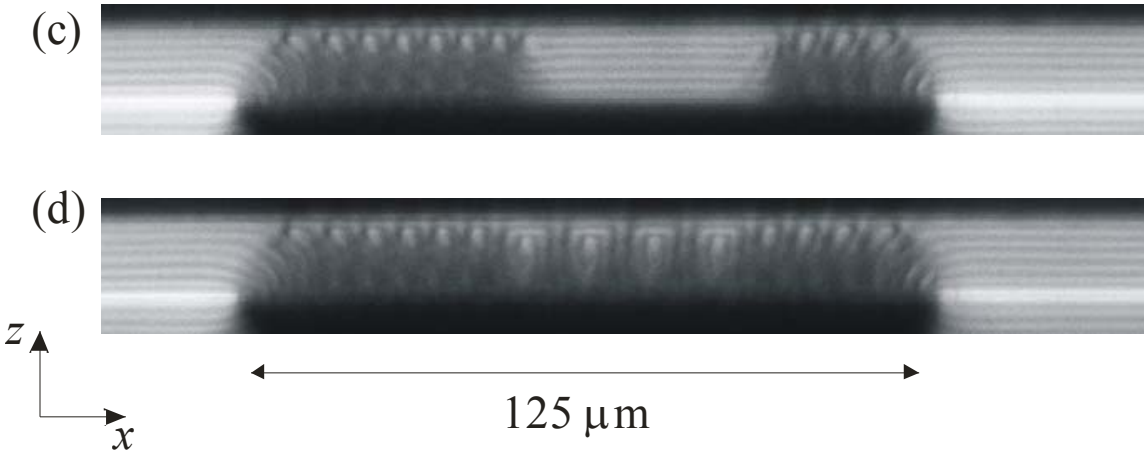

Fig. 5 Cross-section grey-scale images from an $x-z$ plane taken from a MLC-6610* liquid crystal cell at applied peak-to-peak voltages of (a) $0 \mathrm{~V}$, (b) $.50 \mathrm{~V}$, (c) $75 \mathrm{~V}$ and (d) $100 \mathrm{~V}$. The fluorescence intensity is shown as a function of position using a scale varying linearly from black (no fluorescence) to white (maximum recorded fluorescence). The incident light is polarized along the $x$-axis

\subsection{Optical considerations}

As noted previously, an interesting optical effect arises in each of the cross-section images due to the use of optically thick metallic silver as the electrode material. Figure 6 shows an extended $x-z$ cross section collected from an MLC6610* cell where the total depth scanned is over twice the nominal thickness of the liquid crystal layer. The black area that can clearly be seen in the lower central region of the image is in the vicinity of the glass substrate which has no fluorescence at the wavelength of the excitation laser. However, in the adjacent glass regions lying below the silver electrode (which is of a thickness that is less than the distance of one "step" in the $z$-direction) a spatially periodic high fluorescence intensity corresponding to the presence of a cholesteric structure is recorded despite this region being occupied by the plain glass superstrate. This arises from incident excitation light with a focal point below the silver surface being reflected from the metal and focused at a point within the cell. The dye at this point fluoresces and passes back through the system and is then in-focus at the pinhole in the FCPM system. As a result the fluorescence intensity is recorded as originating from the original (but false) focal point.

Figure 6(b) shows a plot of the detected intensity versus focal depth (along the $z$-axis) for points in the $x$-y plane above the silver electrode and above the glass in the electrode gap. The cholesteric pitch of the material can still be clearly distinguished in both sections with the positions of the maxima in the signal collected from the light focused below the electrode region matching those above the plain glass. The oscillations from the fluorescence variation attributed to the cholesteric structure of the liquid crystal appear shallow due to the point spread function of the system, discussed below. There is also a small offset of the fluorescence intensity level due to the fluorescence of the dye along the ordinary axis being non-zero as well as the order parameter of the dye being significantly less than 1 . Analysis of the light collected from the silvered region shows that the data collected from a depth of around $18 \mu \mathrm{m}$ and below is largely a reflection of the first half of the cell with the separation of the maxima exhibiting identical spacings to the separation between the 
corresponding bands above the silver. An additional effect of the silvered region is to prevent the "drop-off" in fluorescence intensity with depth into the sample and instead results in an apparent increase in intensity over a region of around $5 \mu \mathrm{m}$ above the substrate. This is due to light emitted in the positive- $z$ direction being collected as well as light emitted in the negative- $z$ direction which is then reflected by the silver electrode. Interestingly, the intensity of the reflected light at the liquid crystal / silver interface is double the value recorded from the liquid crystal / glass interface. A detailed explanation for this is yet to be determined but it is believed to be related to the influence of the silver layer on the point-spread-function of the system. A subtle "shoulder" can be seen at the same z-position in the data collected above the plain glass layer which can be attributed to a similar (but far less dramatic) effect arising from the refractive index mismatch at the liquid crystal/glass interface. Fluorescence studies using a silvered backplane to enhance backscattered images have previously been used in biological studies using confocal microscopy [22]. It is believed that the enhanced fluorescence intensity produced from the region close to the silver surface may provide a useful additional technique for imaging liquid crystal structures close to an interface [23].
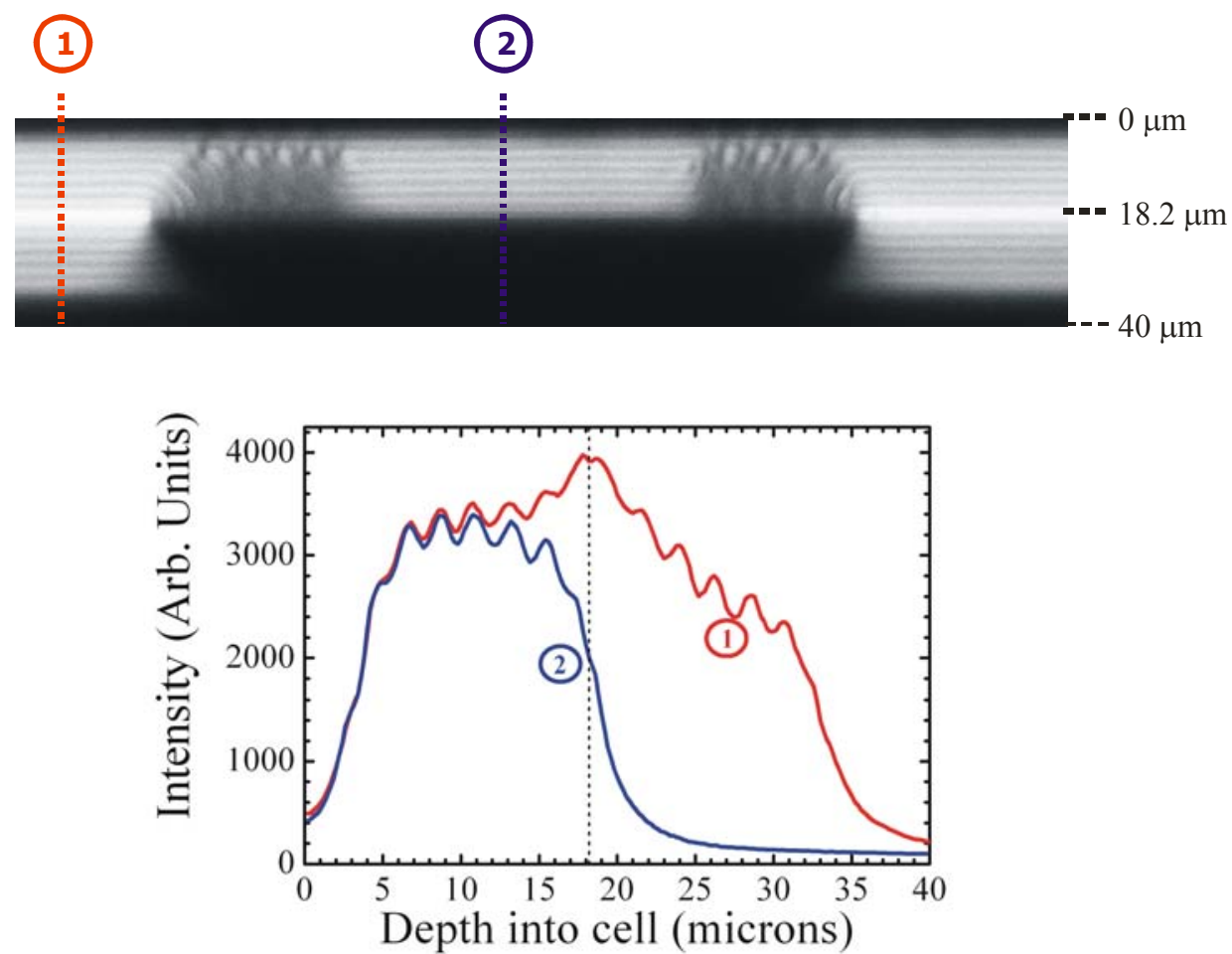

Fig. 6 (a) Cross-section grey-scale image from an $x$-z plane taken from a MLC-6610* liquid crystal cell at applied peak-to-peak voltages $50 \mathrm{~V}$ showing the highly detailed reflected image obtained in the focal region below the silver electrode. (b) Plot of recorded fluorescence intensity versus depth into the cell for the two highlighted regions.

\subsection{Point-spread function}

The shallow appearance of the maxima seen in fig 6(b) are caused by the point-spread function (PSF) of the system [15] which arises due to the refractive-index mismatch of the cover-slip glass and the liquid crystal. In the confocal set-up refraction at the liquid crystal/glass interface results in different rays being focused at different points in the sample, resulting in the focal point being spread out and shifted to a position below the "nominal" focal point that would occur in a perfectly matched system. This results in material outside the intended voxel being illuminated with the excitation light and consequently the fluorescence light produced is able to pass though the pinhole located in front of the intensity detector. Factors dictating the form of the PSF include the numerical aperture of the system, the refractive indices of the 
substrate and liquid crystal, the liquid crystal birefringence, the depth of focus into the sample and the wavelengths of the excitation and emitted light. The result of calculating the PSF for the confocal system used in this study, using a model based on a Debye approximation theory [24] is shown in figure 7. The system was simplified with the liquid crystal approximated as a $20 \mu \mathrm{m}$ thick isotropic layer with a refractive index equal to the mean liquid crystal refractive index $(\mathrm{n}=1.55)$ with the index of the incident glass being $\mathrm{n}=1.52$. Using an excitation wavelength of $488 \mathrm{~nm}$ and NA $=1.4$ calculations at a range of depths into the sample highlight the depth dependent shift between the nominal and actual focal points. In addition, the overall vertical spread of the PSF reaches around $2 \mu \mathrm{m}$ towards the bottom of the sample, although the main maxima of the illumination light is highly localized in a sub-micron region.
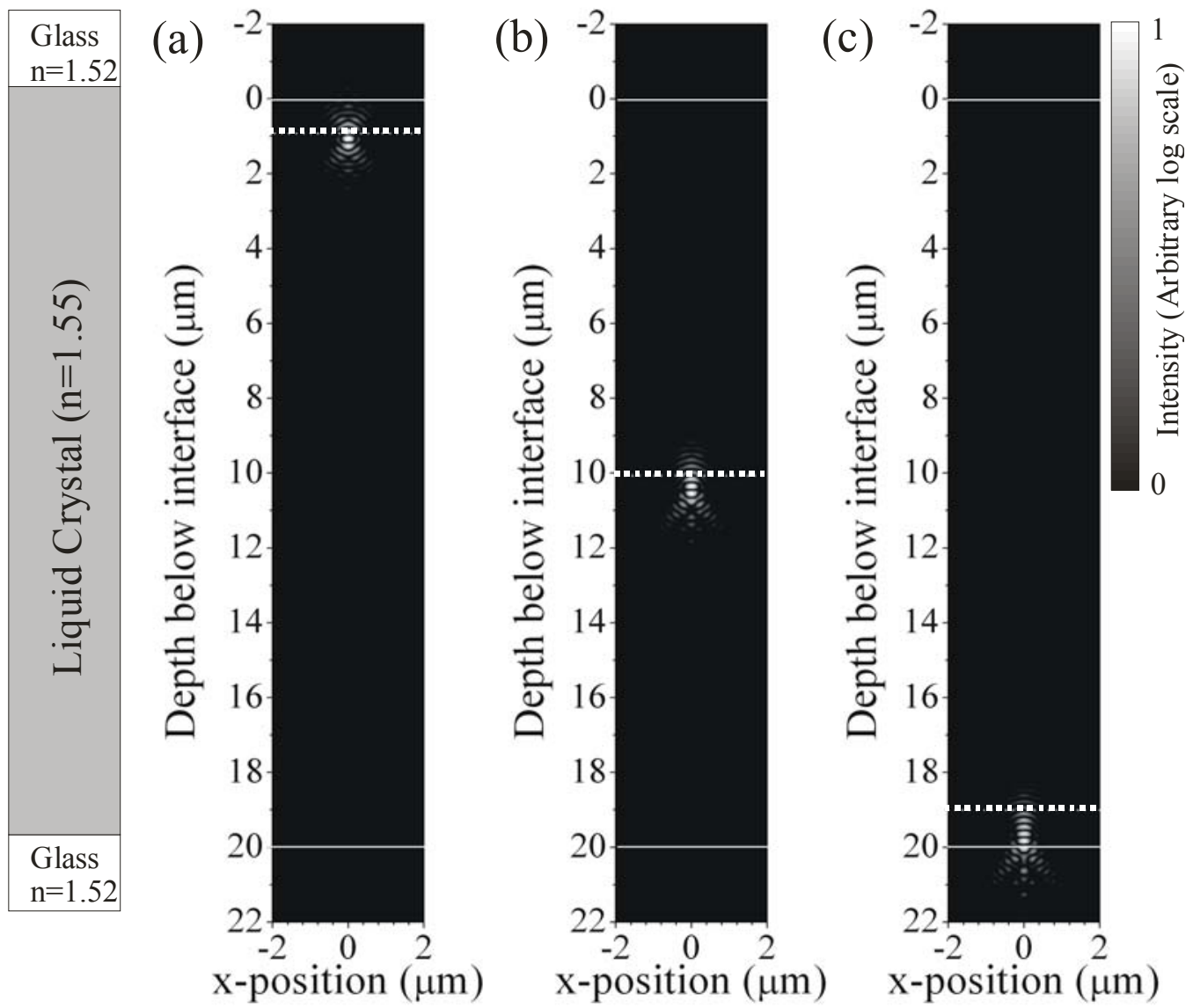

Fig. 7 Modeled point-spread function for a simplified liquid crystal system at focal points of (a) $1 \mu \mathrm{m}$, (b) $10 \mu \mathrm{m}$ and (c) $19 \mu \mathrm{m}$ below the liquid crystal / glass interface. The dashed lines indicate the nominal focal point for a perfectly matched system.

These results illustrate why the $x-z$ cross-section images produced during this study does not show a sharp transition in measured fluorescence intensity as the focal point goes from the glass to the liquid crystal region. The PSF means that regions of the liquid crystal layer are illuminated by the excitation beam when it is actually focused in the nonfluorescent glass, resulting in the gradual increase in fluorescence intensity with depth seen in figure 6(b). Although the PSF for the system does limit the ultimate resolution achievable with fluorescence confocal polarizing microscopy, steps can be taken to deconvolve the fluorescence data collected. In particular, the recorded variation in the intensity with depth at the glass/liquid crystal interface is known to be a convolution of the PSF with the actual top-hat function that occurs at the interface, allowing the PSF to be determined. 


\section{CONCLUSIONS}

This study has shown that fluorescence confocal polarizing microscopy imaging is a powerful technique for imaging liquid crystal structures in 3D. Data collected from cholesteric structures have shown that the application of an in-plane electric field causes a reorientation of the helical axis. The response of the director differs greatly depending on the sign of the dielectric anisotropy; for positive materials the liquid crystal within the gap aligns with the field and stray fields outside the electrode region cause the cholesteric helix to tilt through $90^{\circ}$ and lie parallel to the field direction. For negative materials the helical axis in the region between the electrodes aligns with the field. With the increasing miniaturization of liquid crystal devices and the use of in-plane switching schemes, the effects of stray fields on adjacent pixels is an important issue and must be addressed when designing such systems. During this study, optical effects associated with the FCPM technique have been discussed and a notable enhancement in detected fluorescence intensity in the vicinity of a metal interface has been observed and it is believed that such an effect could be utilized in future FCPM studies. Through the use of a ray-optics model the point-spread function of an FCPM system has also been calculated and the parameters used suggest that micron-scale resolution is achievable, allowing detailed 3D imaging of complex liquid crystal structures.

\section{REFERENCES}

[1] Ohe, M. and Kondo, K., "Electro-optical characteristics and switching behavior of the in-plane switching mode", Applied Physics Letters 67(26), 3895-3897 (1995)

[2] Lee, S.H., Lee, S.L. and Kim H.Y., "Electro-optic characteristics and switching principle of a nematic liquid crystal cell controlled by fringe-field switching," Applied Physics Letters 73(20), 2881-2883 (1998)

[3] Oh-E, M. and Kondo, K. "Advantageous voltage-holding ratio characteristics induced by in-plane electric fields, and the optimization concept of liquid crystals for an in-plane switching electro-optical effect", Liquid Crystals 25(6), 699-709 (1998)

[4] Hong, S.H., et al., "Electro-Optic Characteristic of Fringe-Field Switching Mode Depending on Rubbing Direction, "Japanese Journal of Applied Physics 39(6), L527-L530 (2000)

[5] de Gennes, P.-G. [The physics of liquid crystals] Oxford, Clarendon Press (1974).

[6] Jewell, S.A. and Sambles J.R., "Fully-Leaky Guided Mode Measurement of the Flexoelectric Coefficient $\left(e_{11}+e_{33}\right)$ in a Hybrid Aligned Nematic Liquid Crystal Cell," Mol. Cryst. Liq. Cryst. 401, 181-187 (2003)

[7] Jewell, S.A. and Sambles, J.R., "Optical characterisation of a dual-frequency hybrid aligned nematic liquid crystal cell," Optics Express 13(7), 2627-2632 (2005)

[8] Jewell, S.A. and Sambles, J.R. "Dynamic response of a dual-frequency chiral hybrid aligned nematic liquid crystal cell," Phys. Rev. E 73(1), 011706 (2006)

[9] Jewell, S.A., Taphouse, T.S. and Sambles, J.R. "Rapid switching in a dual-frequency hybrid aligned nematic liquid crystal cell," Applied Physics Letters 87(2), 021106 (2005)

[10] Bryan-Brown, G.P., et al., "Voltage-dependent anchoring of a nematic liquid crystal on a grating surface," Nature 392(6674), 365-367 (1998)

[11] Kitson, S. and Geisow, A., "Controllable alignment of nematic liquid crystals around microscopic posts: Stabilization of multiple states," Applied Physics Letters, 80(19), 3635-3637 (2002)

[12] White, J.G., Amos, W.B. and Fordham, M. "An evaluation of confocal versus conventional imaging in biological structures by fluorescence light-microscopy," Journal of Cell Biology, 105(1), 41-48 (1987)

[13] Dierking, I., "Fluorescence confocal polarizing microscopy: Imaging liquid crystal director fields in three dimensions," Chem. Phys. Chem 2(11), 663-664 (2001)

[14] Smalyukh, II, Shiyanovskii, S.V.and Lavrentovich, O.D., "Three-dimensional imaging of orientational order by fluorescence confocal polarizing microscopy," Chemical Physics Letters 336(1-2), 88-96 (2001)

[15] Pawley, J.B., [Handbook of biological confocal microscopy] New York: Plenum Press (1995)

[16] Smalyukh, II, et al., "Free-standing films of twist grain boundary $T G B(A)$ and UTGB(C*) liquid crystals studied by fluorescence confocal polarizing microscopy," Liquid Crystals 30(8), 877-888 (2003)

[17] Smalyukh, II, et al., "Selective imaging of $3 D$ director fields and study of defects in biaxial smectic A liquid crystals," European Physical Journal E 16(2), 179-191 (2005)

[18] Bramble, J.P., et al., "Observations of focal conic domains in smectic liquid crystals aligned on patterned selfassembled monolayers," Liquid Crystals 34(10), 1137-1143 (2007) 
[19] Smalyukh, II and Lavrentovich, O.D., "Three-dimensional director structures of defects in Grandjean-Cano wedges of cholesteric liquid crystals studied by fluorescence confocal polarizing microscopy," Phys. Rev. E 66(5), 051703 (2002)

[20] Image processing and analysis in Java. [Available from: http://rsb.info.nih.gov/ij/]

[21] Hallam, B.T. et. al., "Quantification of the azimuthal anchoring of a homogeneously aligned nematic liquid crystal using fully-leaky guided modes," Liquid Crystals 26(5), 657-662 (1999)

[22] Gu, M., et al., "Three-dimensional coherent transfer function for reflection confocal microscopy in the presence of refractive-index mismatch," Journal of the Optical Society of America A 18(8), 2002-2008 (2001)

[23] Keith, C.H., Bird, G.J. and Farmer, M.A., "Coherent Backscatter Enhances Reflection Confocal Microscopy," BioImaging Techniques, 25(5), 858-863 (1998).

[24] Egner, A. and Hell, S.W., "Equivalence of the Huygens-Fresnel and Debye approach for the calculation of high aperture point-spread functions in the presence of refractive index mismatch," Journal of Microscopy 193(3), 244249 (1999) 